\title{
Preoperative Korean Shoulder Scoring System Correlates with Preoperative Factors of Rotator Cuff Tears
}

\author{
Eun-Yeol Kim, Byung-Yoon Park, In-Bo Kim \\ Department of Orthopedic Surgery, Busan Bumin Hospital, Busan, Korea
}

Background: The Korean Shoulder Scoring System (KSS) is a reliable and valid procedure for discriminative assessment of the clinical status of patients with rotator cuff tears. This study evaluates the correlation between the preoperative KSS and factors in patients with rotator cuff tears.

Methods: From November 2009 to June 2016, 970 patients who underwent arthroscopic rotator cuff repair were retrospectively evaluated. A total of 490 patients met the study criteria. Preoperative factors included age, sex, symptom duration, mediolateral (ML) and anteroposterior (AP) tear size, acromiohumeral distance (AHD), tangent sign, tendon involvement (type I, supraspinatus; type II, supraspinatus and subscapularis; type III, supraspinatus and infraspinatus; type IV, all 3 tendons), fatty infiltration of rotator cuff muscles (group I, Goutallier stages 0 and 1; group II, Goutallier stages 2, 3, and 4), and KSS.

Results: Old age, ML tear size, and AP tear size negatively correlated with the preoperative KSS $(p<0.001)$. AHD showed a positive correlation with the preoperative KSS $(p<0.001)$. A significantly inferior preoperative KSS was found in females and type III tendon involvement $(p<0.001)$. For supraspinatus and infraspinatus, the preoperative KSS of group II fatty infiltration showed a significantly lower score than group I fatty infiltration $(p<0.05)$.

Conclusions: A relatively lower preoperative KSS was associated with old age, large tear size, narrow AHD, female, type III tendon involvement, and group II fatty infiltration of the supraspinatus and infraspinatus. Our study indicates that preoperative KSS can be a good measurement for the preoperative status of patients with rotator cuff tears.

(Clin Shoulder Elbow 2018;21(1):30-36)

Key Words: Korean Shoulder Scoring System; Rotator cuff tear; Preoperative factors

\section{Introduction}

Rotator cuff tear is one of the most common shoulder disorders, more prevalent in the elderly, causing pain and functional disability including weakness and decreased range of motion (ROM) in the shoulder joint. ${ }^{1,2}$ Along with population aging resulting in a rapid increase in incidence, there have been developments in the diagnostic methods for rotator cuff tear. ${ }^{3,4)}$ Consequently, a commonly accepted treatment for rotator cuff tear, the arthroscopic rotator cuff repair is performed with increasing frequency, with overall satisfactory functional outcomes. ${ }^{5,6)}$ The individual demographics, preoperative level of disability, and the clinical presentation affected the clinical outcomes in patients with rotator cuff tears. ${ }^{7-10)}$ In addition, fatty infiltration and atrophy of rotator cuff muscles, tear size, and tear chronicity are suggested as important factors for rotator cuff healing. ${ }^{11-13)}$

Functional outcomes are evaluated using various outcome assessments; currently, region-specific and condition-specific outcome assessments are used to determine the symptoms and functional outcomes of rotator cuff disorders. The Constant score and the American Shoulder and Elbow Surgeons (ASES) questionnaire for the shoulder are well characterized and accepted in the scientific community. ${ }^{14,15)}$ Although the Constant score is in widespread clinical use particularly in Europe, it is not suit-

Received July 23, 2017. Revised January 9, 2018. Accepted January 15, 2018.

Correspondence to: In-Bo Kim

Department of Orthopedic Surgery, Busan Bumin Hospital, 59 Mandeok-daero, Buk-gu, Busan 46555, Korea

Tel: +82-51-330-3082, Fax: +82-51-330-3075, E-mail: zeusibk@naver.com

IRB approval Busan Bumin Hospital (No. 201707-BM-006).

Financial support: None. Conflict of interests: None. 
able for patients with instability and in research studies because of low inter-rater reliability and concerns regarding validity. The ASES score is a well-tested and helpful combination of self- and clinical assessment for rotator cuff disease; however, caution is required for patients with shoulder instability, since pain comprises $50 \%$ of the overall score. ${ }^{16)}$ The Korean Shoulder Scoring System (KSS) is a useful measurement tool combining both subjective and objective evaluations for shoulder function related to rotator cuff tears. This assessment tool emphasizes the patientoriented self-evaluation to avoid bias produced by observerbased evaluation, and contains the additional muscle endurance domain, which was included only in the KSS. ${ }^{17)}$ The KSS is highly reliable and valid as a discriminative instrument, and shows strong correlation with the ASES and University of California at Los Angeles scoring systems. ${ }^{18)}$

There is little research on which preoperative factors of rotator cuff tears affect the KSS. This study therefore evaluated the correlation between variable preoperative factors affecting patient outcome of rotator cuff tears and the preoperative KSS.

\section{Methods}

\section{Patient Selection}

From November 2009 to June 2016, 970 patients underwent arthroscopic repair of rotator cuff tears, performed by the senior author. Only patients with a full-thickness tear of the supraspinatus tendon, with or without a combined full thickness tear of any other rotator cuff tendon, were included. Exclusion criteria included 197 cases of partial thickness tear of any other rotator cuff tendons, 17 ineligible magnetic resonance imagings (MRIs), 30 revision surgeries, 59 cases of preoperative stiffness, 4 isolated subscapularis (SSc) repair, and 173 cases of unmeasured KSS. Finally, 490 patients were retrospectively reviewed for the collected data.

\section{Clinical Assessment and Radiographic Evaluation}

Before undergoing arthroscopic rotator cuff repair, patients were assessed by MRI and their KSS was measured. All MRI studies were performed with the Magnetom Symphony 1.5T MRI system (Siemen, Erlangen, Germany) and the Signa EXCITE 1.5T MRI system (GE, Milwaukee, WI, USA). The preoperative MRI scans were reviewed by 2 experienced radiologists. The KSS included both subjective outcomes such as function (30 points), pain (20 points), and satisfaction (10 points), and objective outcomes such as active ROM (20 points) and muscle power consisting of strength (10 points) and endurance (10 points). To analyze these factors, we considered the following parameters: age, sex, symptom duration before surgery, mediolateral $(\mathrm{ML})$ and anteroposterior $(\mathrm{AP})$ tear size of the tendon, acromiohumeral distance (AHD), tangent sign, tendon involvement, and degree of fatty degeneration of rotator cuff muscles. The ML and
AP size of tendon tears (in millimeters) were evaluated on T2weighted images in oblique coronal and oblique sagittal MRIs, as described by Davidson et al. ${ }^{19)}$ According to the tendon involvement, the pattern of tendon tear were divided into 4 categories: tear of supraspinatus (type I), tears of supraspinatus and SSc (type II), tears of supraspinatus and infraspinatus (IST; type III), and tear of all 3 tendons (type IV). ${ }^{10)}$ Muscle atrophy of the supraspinatus was evaluated by use of the tangent sign on the most lateral T2weighted oblique sagittal MRI in which the scapular spine contacted the scapular body. The tangent sign was considered positive when the superior border of the supraspinatus muscle belly was inferior in relation to the line tangential to the coracoids and scapular spine. ${ }^{20)}$ Fatty degeneration of the rotator cuff muscles was measured using preoperative MRI according to the classification established by Goutallier et al. ${ }^{21)}$ and modified by Fuchs et al. ${ }^{22)}$ For further statistical analysis, the severity of rotator cuff fatty infiltration was divided into 2 groups: stages 0 and 1 (group I), and stages 2,3 , and 4 (group II). ${ }^{10)}$

\section{Statistical Analysis}

Pearson correlation coefficient (r) was calculated to measure the degree of correlation between KSS and continuous variables such as age, symptom duration before surgery, ML and AP tear sizes, and AHD. These correlation coefficients ranged from - 1 to 1 , where 1 indicates a positive relationship and -1 a negative relationship. Agreements are considered to be almost perfect for correlation coefficients from 0.81 to 1.00 , excellent for 0.61 to 0.80 , good to moderate for 0.41 to 0.61 , fair for 0.21 to 0.40 , and poor for 0.00 to $0.20{ }^{23)}$ Chi-square test or Fisher's exact test was performed to compare KSS in nominal variables such as sex and tangent sign. One-way analysis of variance was used to determine a possible relationship between KSS, and tendon involvement and fatty infiltration. Scheffe or Dunnett's T post hoc test was used for multiple comparison among groups of tendon involvement and fatty infiltration, according to the homogeneity of variance. SPSS-IBM software package ver. 20.0 (IBM Co., Armonk, NY, USA) was used for all statistical analyses, and the $\alpha$ level was set at 0.05 .

\section{Results}

The demographic and radiographic data are summarized in Table 1. Our patients included 247 males and 243 females, with a mean age of $59.2 \pm 7.8$ years. The average symptomatic duration was $16.2 \pm 34.8$ months. Preoperative mean KSS was 65.1 $\pm 14.5$

\section{Age, Symptom Duration, Tear Size, Acromiohumeral Distance}

Old age negatively correlated with the preoperative KSS ( $r=$ $-0.272, p<0.001)$. Age also demonstrated a significantly nega- 
Table 1. Demographic Data for Clinical Variables $(n=490)$

\begin{tabular}{|c|c|}
\hline Variable & Value \\
\hline Age (yr) & $59.2 \pm 7.8(37.0-83.0)$ \\
\hline Sex (male:female) & $247: 243$ \\
\hline Symptom duration (mo) & $16.2 \pm 34.8(1-480)$ \\
\hline Preoperative Korean Shoulder Scoring System & $65.1 \pm 14.5(6-93)$ \\
\hline Function & $20.1 \pm 4.4(0-30)$ \\
\hline Pain & $12.4 \pm 3.6(0-20)$ \\
\hline Satisfaction & $4.2 \pm 2.1(0-20)$ \\
\hline Range of motion & $14.4 \pm 4.0(0-20)$ \\
\hline Strength & $6.9 \pm 2.1(0-10)$ \\
\hline Endurance & $6.5 \pm 4.5(0-18)$ \\
\hline \multicolumn{2}{|l|}{ Tear size $(\mathrm{mm})$} \\
\hline Mediolateral & $23.8 \pm 11.9(2.0-70.0)$ \\
\hline Anteroposterior & $18.3 \pm 11.3(2.7-70.0)$ \\
\hline Acromiohumeral distance (mm) & $9.0 \pm 1.6(3.7-13.9)$ \\
\hline \multicolumn{2}{|l|}{ Tendon involvement } \\
\hline Supraspinatus & 430 \\
\hline Supraspinatus+subscapularis & 28 \\
\hline Suparspintus+infraspinatuss & 32 \\
\hline All 3 tendons & 0 \\
\hline Tangent sign & 12 \\
\hline \multicolumn{2}{|c|}{ Fatty infiltration, subscapularis/supraspinatus/infraspintus/teres minor (n) } \\
\hline 0 & $149 / 82 / 50 / 211$ \\
\hline 1 & $308 / 348 / 300 / 252$ \\
\hline 2 & $30 / 55 / 125 / 24$ \\
\hline 3 & $3 / 4 / 8 / 0$ \\
\hline 4 & $0 / 1 / 7 / 3$ \\
\hline
\end{tabular}

Values are presented as mean \pm standard deviation (range) or number only.

tive correlation with function $(r=-0.183, p<0.001)$, ROM $(r=$ -0.295, $p<0.001)$, strength $(r=-0.219, p<0.001)$, and endurance $(r=-0.214, p<0.001)$ of KSS domains.

Symptom duration did not correlate with preoperative KSS, but significantly correlated with pain of KSS domains ( $r=-0.092$, $p=0.041$ ), and a longer symptom duration had significantly lower score in pain.

The mean ML tear size was $23.8 \pm 11.9 \mathrm{~mm}$, and the mean AP tear size was $18.3 \pm 11.3 \mathrm{~mm}$; both were negatively correlated with the preoperative KSS $(r=-0.228, p<0.001$; and $r=$ -0.193, $p<0.001$, respectively). Tear size did not correlate with pain and satisfaction of KSS domains.

The AHD demonstrated significantly positive correlation with preoperative KSS $(r=0.171, p<0.001)$. AHD demonstrated a significant correlation with function $(r=0.129, p=0.004)$, ROM $(r=0.134, p=0.003)$, strength $(r=0.207, p<0.001)$, and endurance $(r=0.218, p<0.001)$ of KSS domains, but did not correlate with pain and satisfaction (Table 2).

\section{Sex, Tangent Sign}

On preoperative KSS, the scores of female patients (61.9 $\pm 15.2)$ were significantly inferior compared to male patients $(68.2 \pm 13.1)(p<0.001)$. Females showed a significantly inferior score than males with regards to function $(p=0.001)$, strength $(p<0.001)$, and endurance $(p<0.001)$ of the KSS domains. However, the gender did not correlate with pain, satisfaction, and ROM of KSS domains.

Tangent sign did not correlate with preoperative KSS or any of the KSS domains (Table 2).

\section{Tendon Involvement}

With respect to tendon involvement, the mean preoperative KSS were as follow: $66.1 \pm 13.6$ (type I), 58.7 \pm 20.6 (type II), and $57.1 \pm 17.0$ (type III). Patients with type III tendon involvement $(57.1 \pm 17.0)$ showed a significantly inferior KSS than type I tendon involvement $(66.1 \pm 13.6)(p<0.001)$. Also, patients with type III tendon involvement showed a significantly inferior score on function $(p=0.046), \operatorname{ROM}(p<0.001)$, and endurance $(p=0.010)$ of KSS domains than type I tendon involvement. Especially, tendon involvement did not correlate with pain, satisfaction, and strength of KSS domains (Table 3).

\section{Fatty Infiltration}

For Simple Shoulder Test (SST), the group II fatty infiltration $(59.8 \pm 14.7)$ showed a significantly lower score than the group I fatty infiltration $(65.8 \pm 14.4)$ on preoperative KSS $(p=0.003)$, which was significantly different between groups I and II fatty infiltration in function $(p=0.013), \operatorname{ROM}(p=0.016)$, strength $(p=0.022)$, and endurance $(p=0.007)$ of the KSS domains, but not significantly different in pain and satisfaction.

For IST, the group II fatty infiltration $(62.9 \pm 15.5)$ showed a significantly lower score than the group I fatty infiltration (66.0 \pm 14.1) on preoperative KSS ( $p=0.038)$, and was significantly different between groups I and II fatty infiltration in $\operatorname{ROM}(p=0.011)$ domain and total $(p=0.038)$, but not significant different in the other domains.

For SSc, the preoperative KSS was significantly different between the groups I and II fatty infiltration only in the pain domain $(p=0.046)$, with no significant differences observed in the other domains.

For teres minor $(\mathrm{Tm})$, the preoperative KSS was significantly different between groups I and II fatty infiltration in function $(p=0.030)$ and ROM $(p=0.004)$ of the KSS domains, with no significant differences in the other domains.

Especially, the fatty infilitration of IST, SSc, and Tm had no correlation with strength and endurance (Table 4). 
Table 2. Correlation between Various Factors and the Preoperative Korean Shoulder Scoring System

\begin{tabular}{|c|c|c|c|c|c|c|c|}
\hline Variable & Function & Pain & Satisfaction & ROM & Strength & Endurance & Total \\
\hline \multicolumn{8}{|l|}{ Age } \\
\hline Correlation (r) & -0.183 & -0.066 & -0.029 & -0.295 & -0.219 & -0.214 & -0.272 \\
\hline$p$-value ${ }^{*}$ & $<0.001^{*}$ & 0.145 & 0.517 & $<0.001^{*}$ & $<0.001^{*}$ & $<0.001^{\star}$ & $<0.001^{\star}$ \\
\hline \multicolumn{8}{|l|}{ Symptom duration } \\
\hline Correlation (r) & 0.039 & -0.092 & -0.061 & 0.044 & 0.077 & 0.076 & 0.025 \\
\hline$p$-value & 0.390 & $0.041^{*}$ & 0.180 & 0.334 & 0.088 & 0.091 & 0.575 \\
\hline \multicolumn{8}{|l|}{ ML tear size } \\
\hline Correlation (r) & -0.129 & -0.040 & -0.052 & -0.233 & -0.256 & -0.198 & -0.228 \\
\hline$p$-value & $0.004^{*}$ & 0.375 & 0.248 & $<0.001^{*}$ & $<0.001^{*}$ & $<0.001^{*}$ & $<0.001^{\star}$ \\
\hline \multicolumn{8}{|l|}{ AP tear size } \\
\hline Correlation (r) & -0.117 & 0.028 & -0.028 & -0.234 & -0.202 & -0.166 & -0.193 \\
\hline$p$-value & $0.010^{*}$ & 0.538 & 0.530 & $<0.001^{\star}$ & $<0.001^{\star}$ & $<0.001^{*}$ & $<0.001^{\star}$ \\
\hline \multicolumn{8}{|l|}{ AHD } \\
\hline Correlation (r) & 0.129 & 0.002 & 0.019 & 0.134 & 0.207 & 0.218 & 0.171 \\
\hline$p$-value & $0.004^{*}$ & 0.970 & 0.680 & $0.003^{*}$ & $<0.001^{\star}$ & $<0.001^{*}$ & $<0.001^{\star}$ \\
\hline \multicolumn{8}{|l|}{$\operatorname{Sex}^{*}$} \\
\hline Male & $20.7 \pm 4.0$ & $12.7 \pm 3.4$ & $4.3 \pm 1.9$ & $14.7 \pm 3.8$ & $7.3 \pm 2.0$ & $7.9 \pm 3.9$ & $68.2 \pm 13.1$ \\
\hline Female & $19.4 \pm 4.8$ & $12.1 \pm 3.8$ & $4.2 \pm 2.2$ & $14.1 \pm 4.2$ & $6.4 \pm 2.1$ & $5.2 \pm 4.6$ & $61.9 \pm 15.2$ \\
\hline$p$-value & $0.001^{\dagger}$ & 0.081 & 0.353 & 0.117 & $<0.001^{\dagger}$ & $<0.001^{\dagger}$ & $<0.001^{\dagger}$ \\
\hline \multicolumn{8}{|l|}{ Tangent sign ${ }^{*}$} \\
\hline No & $20.1 \pm 4.5$ & $12.4 \pm 3.6$ & $4.2 \pm 2.1$ & $14.5 \pm 4.0$ & $6.9 \pm 2.0$ & $6.6 \pm 4.5$ & $65.2 \pm 14.4$ \\
\hline Yes & $20.4 \pm 4.3$ & $12.5 \pm 3.4$ & $4.7 \pm 1.8$ & $13.3 \pm 4.9$ & $5.5 \pm 3.6$ & $5.3 \pm 4.8$ & $62.5 \pm 20.1$ \\
\hline$p$-value & 0.806 & 0.945 & 0.480 & 0.344 & 0.213 & 0.342 & 0.533 \\
\hline
\end{tabular}

ROM: range of motion, ML: mediolateral, AP: anteroposterior, AHD: acromiohumeral distance.

${ }^{*}$ Statistically significant difference $(p<0.05)$.

${ }^{\dagger}$ Statistical significances $(p<0.05)$ were tested by chi-square or Fisher's exact test.

${ }^{*}$ Mean \pm standard deviation.

Table 3. Correlation between Tendon Involvement and the Preoperative Korean Shoulder Scoring System

\begin{tabular}{|c|c|c|c|c|c|c|c|}
\hline Variable & Function & Pain & Satisfaction & ROM & Strength & Endurance & Total \\
\hline Type I & $20.3 \pm 4.3$ & $12.5 \pm 3.5$ & $4.3 \pm 2.1$ & $14.8 \pm 3.7$ & $6.9 \pm 2.1$ & $6.8 \pm 4.4$ & $66.1 \pm 13.6$ \\
\hline $\mathrm{T}^{\dagger}$ & $\mathrm{a}$ & a & $\mathrm{a}$ & $\mathrm{a}$ & $\mathrm{a}$ & $\mathrm{a}$ & $\mathrm{a}$ \\
\hline Type II & $19.7 \pm 6.4$ & $10.9 \pm 4.5$ & $4.0 \pm 2.2$ & $12.2 \pm 5.3$ & $6.4 \pm 2.3$ & $5.5 \pm 4.8$ & $58.7 \pm 20.6$ \\
\hline $\mathrm{T}^{\dagger}$ & $\mathrm{a}, \mathrm{b}$ & $\mathrm{a}, \mathrm{b}$ & $\mathrm{a}$ & $\mathrm{a}, \mathrm{b}$ & a & $\mathrm{a}, \mathrm{b}$ & $\mathrm{a}, \mathrm{b}$ \\
\hline Type III & $18.3 \pm 4.6$ & $12.2 \pm 3.8$ & $3.9 \pm 2.0$ & $11.9 \pm 5.4$ & $6.2 \pm 1.9$ & $4.5 \pm 4.5$ & $57.1 \pm 17.0$ \\
\hline $\mathrm{T}^{\dagger}$ & $\mathrm{b}$ & $\mathrm{b}$ & $\mathrm{a}$ & b & $\mathrm{a}$ & b & b \\
\hline$p$-value & $0.046^{*}$ & 0.061 & 0.457 & $<0.001^{\star}$ & 0.050 & $0.010^{*}$ & $<0.001^{*}$ \\
\hline
\end{tabular}

Values are presented as mean \pm standard deviation.

ROM: range of motion, Type I: supraspinatus, Type II: supraspinatus and subscapularis, Type III: supraspinatus and infraspinatus.

${ }^{*}$ Statistical significances $(p<0.05)$ were tested by One way analysis of variances among groups.

${ }^{\dagger}$ The same letters indicate non-significant difference between groups based on Scheffe or Dunnett's T multiple comparison test according to the homogeneity of variance. 
Table 4. Correlation between Fatty Infiltration and the Preoperative Korean Shoulder Scoring System

\begin{tabular}{|c|c|c|c|c|c|c|c|}
\hline Variable & Function & Pain & Satisfaction & ROM & Strength & Endurance & Total \\
\hline \multicolumn{8}{|l|}{ Subscapularis } \\
\hline Group I & $20.1 \pm 4.4$ & $12.5 \pm 3.6$ & $4.2 \pm 2.1$ & $14.4 \pm 4.0$ & $6.9 \pm 2.1$ & $6.6 \pm 4.5$ & $65.2 \pm 14.3$ \\
\hline Group II & $20.1 \pm 5.4$ & $11.2 \pm 3.3$ & $4.4 \pm 2.0$ & $14.5 \pm 4.6$ & $6.9 \pm 1.8$ & $6.1 \pm 4.5$ & $63.8 \pm 17.1$ \\
\hline$p$-value & 0.954 & $0.046^{*}$ & 0.611 & 0.965 & 0.877 & 0.573 & 0.595 \\
\hline \multicolumn{8}{|l|}{ Supraspinatus } \\
\hline Group I & $20.3 \pm 4.5$ & $12.5 \pm 3.6$ & $4.3 \pm 2.1$ & $14.6 \pm 4.0$ & $6.9 \pm 2.1$ & $6.8 \pm 4.4$ & $65.8 \pm 14.4$ \\
\hline Group II & $18.8 \pm 4.1$ & $12.0 \pm 3.6$ & $3.9 \pm 1.9$ & $13.3 \pm 4.4$ & $6.3 \pm 2.1$ & $5.0 \pm 4.8$ & $59.8 \pm 14.7$ \\
\hline$p$-value & $0.013^{*}$ & 0.330 & 0.119 & $0.016^{*}$ & $0.022^{*}$ & $0.007^{*}$ & $0.003^{*}$ \\
\hline \multicolumn{8}{|l|}{ Infraspinatus } \\
\hline Group I & $20.1 \pm 4.4$ & $12.5 \pm 3.5$ & $4.3 \pm 2.1$ & $14.7 \pm 3.8$ & $7.0 \pm 2.0$ & $6.8 \pm 4.4$ & $66.0 \pm 14.1$ \\
\hline Group II & $20.0 \pm 4.6$ & $12.3 \pm 3.9$ & $4.1 \pm 1.9$ & $13.6 \pm 4.5$ & $6.6 \pm 2.2$ & $6.0 \pm 4.6$ & $62.9 \pm 15.5$ \\
\hline$p$-value & 0.744 & 0.492 & 0.227 & $0.011^{*}$ & 0.055 & 0.096 & $0.038^{*}$ \\
\hline \multicolumn{8}{|l|}{ Teres minor } \\
\hline Group I & $20.2 \pm 4.4$ & $12.4 \pm 3.6$ & $4.3 \pm 2.1$ & $14.6 \pm 4.0$ & $6.9 \pm 2.1$ & $6.6 \pm 4.4$ & $65.4 \pm 14.3$ \\
\hline Group II & $18.3 \pm 4.8$ & $12.2 \pm 3.8$ & $4.1 \pm 1.8$ & $12.3 \pm 4.2$ & $6.3 \pm 2.3$ & $5.6 \pm 4.9$ & $60.1 \pm 17.1$ \\
\hline$p$-value & $0.030^{*}$ & 0.762 & 0.733 & $0.004^{*}$ & 0.150 & 0.291 & 0.067 \\
\hline
\end{tabular}

Values are presented as mean \pm standard deviation.

ROM: range of motion, Group I: Goutallier stages 0 and 1, Group II: Goutallier stages 2, 3, and 4.

${ }^{*}$ Statistical significances $(p<0.05)$ were tested by independent $\mathrm{t}$-test between groups.

\section{Discussion}

The KSS may have been valid as a discriminative instrument for shoulder function related to rotator cuff tears, but little is known as to what affects the KSS. Therefore, we identified the factors possibly affecting KSS, and tried to evaluate the usefulness of KSS in assessing preoperative rotator cuff tear patients. Among the various preoperative factors investigated in this study, a significantly lower preoperative KSS was associated with older age, female, large tear size, narrow AHD, type III tendon involvement, and group II fatty infiltration.

A few studies have identified the association between age and shoulder scoring system. Godfrey et al. ${ }^{24)}$ suggested that since the SST can be affected by age in healthy people, hence a lower score is to be expected in older patients. Oh et al. ${ }^{25)}$ demonstrated older patients had poor results on SST scoring. In addition, the relationship between age and tear size has also been previously reported. Yamaguchi et al. ${ }^{26,27)}$ determined that the natural progression of rotator cuff tear occurs over time, and showed a high correlation with advanced age. Nho et al. ${ }^{28)}$ confirmed that age was a significant independent risk factor for tendon defect after arthroscopic rotator cuff repair, and increased 1.08 times for every additional year. Based on previous studies, elderly patients tend to show an increase in tear size. Our study found older patients showed a significantly lower preoperative KSS. Also, in the older patients with reduced muscle strength due to sarcopenia resulting in reduced activity, lower was the score for function, strength, and endurance of KSS. If the elderly patient had extensive tendon rupture and severe fatty degeneration, it was difficult to assess the reasons for lower KSS. Therefore, the age may be obviated by other powerful affecting factors such as larger tear size or higher stage fatty infiltration. Further analysis of the KSS with normal shoulder values and normalization values related to age are therefore required.

Several studies reported that tear size correlated with the scores of shoulder scoring systems. Shin et al. ${ }^{18)}$ reported that the preoperative KSS scores of patients with small to medium sized tears were significantly higher than those for patients with large to massive sized tears. According to the study by Park et al., ${ }^{12)}$ the size of the tear correlated with the ASES score, and further demonstrated that the tear size in the AP direction was a preoperative factor affecting rotator cuff healing. Our result also determined that the larger tear size showed a significant inferior score in the preoperative KSS. Interestingly, both the ML tear size and the AP tear size were significantly associated with preoperative KSS.

Most studies to date have been based on whether or not the number of torn tendons affects the clinical outcome. Nho et al. ${ }^{28)}$ reported that number of tendons involved was a significant prognostic factor after rotator cuff repair. According to the study by Shin et al.., ${ }^{18)}$ the preoperative KSS scores of patients with multiple torn tendons were significantly lower than those for 
patients with a single torn tendon. We similarly found that additional tears of other tendons along with tears of the supraspinatus tendon showed a tendency to decrease the preoperative KSS, although it was statistically not significant. Moreover, patients with type III tendon involvement showed a significantly inferior KSS score than patients with type I tendon involvement. This suggests that the clinical feature in the rotator cuff tears may be related to the tendon involvement with regards to IST involvement rather than just the tendon tear. Unlike a previous study by Shin et al., ${ }^{18)}$ our study demonstrated that the preoperative factor affecting preoperative KSS is with respect to which tendon is torn, and not just the number of the tendon(s) involved.

Fatty infiltration is recognized as a factor affecting the status of rotator cuff disease and postoperative clinical outcomes. Gladstone et al. ${ }^{29)}$ reported that atrophy and fatty infiltration of the IST muscle were the only independent predictors of the ASES and Constant scores. Shin et al. ${ }^{18)}$ reported that a significantly inferior score in the preoperative KSS response was observed in patients with preoperative fatty degeneration greater than or equal to stage 3 of the Goutallier grading system. We similarly observed a close correlation between fatty infiltration of the cuff muscle and the preoperative KSS scores. However, KSS was significantly lower at stage 3 or higher fatty infiltration in the other study by Shin et al., ${ }^{18)}$ whereas KSS was significantly lower at stage 2 or higher fatty infiltration in our study. Although there is a discrepancy in the specific details between both studies, the common point remains that fatty infiltration affects the preoperative KSS.

An interesting finding of our study was the correlation between various preoperative factors and each domain of preoperative KSS. The function domain is affected by age, sex, tear size, AHD, tendon involvement, and fatty infiltration. Pain and satisfaction domains are not affected by preoperative factors. ROM, strength, and endurance domains are affected by age, sex, tear size, AHD, tendon involvement, and fatty infiltration to affected KSS in general. However, the sex does not correlate with the ROM domain, and tendon involvement did not correlate with strength domain. Age, tear size, AHD, sex, tendon involvement, and fatty infiltration of rotator cuff muscle (except SSc) did not correlate with pain and satisfaction of objective outcomes in KSS. Age, tear size, AHD, sex, and fatty infiltration of SST correlate with strength and endurance of subjective outcomes in KSS. We thereby suggest that most preoperative factors having a correlation with the preoperative KSS mainly affect the overall subjective outcome of KSS. Especially, fatty infiltration of rotator cuff muscles (except SST) did not correlate with strength and endurance. This indicates that fatty infiltration of SST is an important factor affecting the muscle tone. It would be interesting to further study the relationship between each of the preoperative factors affecting KSS.

In the current study, age, tear size, AHD, sex, tendon involve- ment, and fatty infiltration of SST and IST were thought to be important factors affecting preoperative KSS score.

There were some limitations to our study. First, since no patient was assessed as tendon involvement type IV, this category could not be included in the analysis. Second, the limitation of this study was that arbitrary grouping was performed due to the small number of patients in some groups. If the groups with smaller patient numbers were excluded, several groups such as Goutallier stage 3 of rotator cuff muscles (SSc, SST, IST, Tm) in the tear group (3/4/8/0 patients) and Goutallier stage 4 of the rotator cuff muscles in the tear group (0/1/7/3 patients) would also be excluded, making it difficult to analyze the data. Therefore, we chose to combine Goutallier stages 0 and 1 into one group, and Goutallier stages 2, 3 and 4 into another. Third, our study was a retrospective study with inherent limitations.

\section{Conclusion}

A relatively lower preoperative KSS score was associated with old age, large tear size, narrow AHD, female gender, type III tendon involvement, and group II fatty infiltration of the SST and IST. An inferior preoperative score in function, ROM, and endurance domains was associated with old age, large tear size, narrow AHD, female gender, type III tendon involvement, and group II fatty infiltration of the SST and IST. We conclude that KSS is a good measurement to identify various factors affecting the clinical status of patients with rotator cuff tear before surgery.

\section{References}

1. Fehringer EV, Sun J, VanOeveren LS, Keller BK, Matsen FA 3rd. Full-thickness rotator cuff tear prevalence and correlation with function and co-morbidities in patients sixty-five years and older. J Shoulder Elbow Surg. 2008;17(6):881-5.

2. Galatz LM, Ball CM, Teefey SA, Middleton WD, Yamaguchi K. The outcome and repair integrity of completely arthroscopically repaired large and massive rotator cuff tears. J Bone Joint Surg Am. 2004;86(2):219-24.

3. Needell SD, Zlatkin MB, Sher JS, Murphy BJ, Uribe JW. MR imaging of the rotator cuff: peritendinous and bone abnormalities in an asymptomatic population. AJR Am J Roentgenol. 1996;166(4):863-7.

4. Tashjian RZ. Epidemiology, natural history, and indications for treatment of rotator cuff tears. Clin Sports Med. 2012;31(4):589-604.

5. Jost B, Pfirrmann CW, Gerber C, Switzerland Z. Clinical outcome after structural failure of rotator cuff repairs. J Bone Joint Surg Am. 2000;82(3):304-14.

6. Anderson K, Boothby M, Aschenbrener D, van Holsbeeck M. Outcome and structural integrity after arthroscopic rotator cuff repair using 2 rows of fixation: minimum 2-year follow-up. 
Am J Sports Med. 2006;34(12):1899-905.

7. Cho NS, Rhee YG. The factors affecting the clinical outcome and integrity of arthroscopically repaired rotator cuff tears of the shoulder. Clin Orthop Surg. 2009;1(2):96-104.

8. Oh JH, Kim SH, Kim KH, Oh $\mathrm{CH}$, Gong HS. Modified impingement test can predict the level of pain reduction after rotator cuff repair. Am J Sports Med. 2010;38(7):1383-8.

9. Gulotta LV, Nho SJ, Dodson CC, Adler RS, Altchek DW, MacGillivray JD; HSS Arthroscopic Rotator Cuff Registry. Prospective evaluation of arthroscopic rotator cuff repairs at 5 years: part II--prognostic factors for clinical and radiographic outcomes. J Shoulder Elbow Surg. 2011;20(6):941-6.

10. Kim IB, Kim MW. Risk factors for retear after arthroscopic repair of full-thickness rotator cuff tears using the suture bridge technique: classification system. Arthroscopy. 2016;32(11):2191-200.

11. Boileau P, Brassart N, Watkinson DJ, Carles M, Hatzidakis AM, Krishnan SG. Arthroscopic repair of full-thickness tears of the supraspinatus: does the tendon really heal? J Bone Joint Surg Am. 2005;87(6):1229-40.

12. Park JS, Park HJ, Kim SH, Oh JH. Prognostic factors affecting rotator cuff healing after arthroscopic repair in small to medium-sized tears. Am J Sports Med. 2015;43(10):2386-92.

13. Tashjian RZ, Hollins AM, Kim HM, et al. Factors affecting healing rates after arthroscopic double-row rotator cuff repair. Am J Sports Med. 2010;38(12):2435-42.

14. Constant CR, Gerber C, Emery RJ, Søjbjerg JO, Gohlke F, Boileau P. A review of the Constant score: modifications and guidelines for its use. J Shoulder Elbow Surg. 2008;17(2):35561.

15. Richards RR, An KN, Bigliani LU, et al. A standardized method for the assessment of shoulder function. J Shoulder Elbow Surg. 1994;3(6):347-52.

16. Kirkley A, Griffin S, Dainty K. Scoring systems for the functional assessment of the shoulder. Arthroscopy. 2003;19(10):110920.

17. Tae SK, Rhee YG, Park TS, et al. The development and validation of an appraisal method for rotator cuff disorders: the Korean Shoulder Scoring System. J Shoulder Elbow Surg. 2009;18(5):689-96.

18. Shin SJ, Lee J, Ko YW, Park MG. Evaluation of rotator cuff repair using Korean Shoulder Scoring System. Clin Should Elbow. 2015;18(4):206-10.

19. Davidson JF, Burkhart SS, Richards DP, Campbell SE. Use of preoperative magnetic resonance imaging to predict rotator cuff tear pattern and method of repair. Arthroscopy. 2005;21(12):1428.

20. Mellado JM, Calmet J, Olona M, et al. Surgically repaired massive rotator cuff tears: MRI of tendon integrity, muscle fatty degeneration, and muscle atrophy correlated with intraoperative and clinical findings. AJR Am J Roentgenol. 2005;184(5):145663.

21. Goutallier D, Postel JM, Bernageau J, Lavau L, Voisin MC. Fatty muscle degeneration in cuff ruptures. Pre- and postoperative evaluation by CT scan. Clin Orthop Relat Res. 1994;(304):7883.

22. Fuchs B, Weishaupt D, Zanetti M, Hodler J, Gerber C. Fatty degeneration of the muscles of the rotator cuff: assessment by computed tomography versus magnetic resonance imaging. J Shoulder Elbow Surg. 1999;8(6):599-605.

23. Landis JR, Koch GG. The measurement of observer agreement for categorical data. Biometrics. 1977;33(1):159-74.

24. Godfrey J, Hamman R, Lowenstein S, Briggs K, Kocher M. Reliability, validity, and responsiveness of the simple shoulder test: psychometric properties by age and injury type. J Shoulder Elbow Surg. 2007;16(3):260-7.

25. Oh JH, Kim SH, Ji HM, Jo KH, Bin SW, Gong HS. Prognostic factors affecting anatomic outcome of rotator cuff repair and correlation with functional outcome. Arthroscopy. 2009;25(1):30-9.

26. Yamaguchi K, Ditsios K, Middleton WD, Hildebolt CF, Galatz LM, Teefey SA. The demographic and morphological features of rotator cuff disease. A comparison of asymptomatic and symptomatic shoulders. J Bone Joint Surg Am. 2006;88(8):1699-704.

27. Yamaguchi K, Tetro AM, Blam O, Evanoff BA, Teefey SA, Middleton WD. Natural history of asymptomatic rotator cuff tears: a longitudinal analysis of asymptomatic tears detected sonographically. J Shoulder Elbow Surg. 2001;10(3):199-203.

28. Nho SJ, Brown BS, Lyman S, Adler RS, Altchek DW, MacGillivray JD. Prospective analysis of arthroscopic rotator cuff repair: prognostic factors affecting clinical and ultrasound outcome. J Shoulder Elbow Surg. 2009;18(1):13-20.

29. Gladstone JN, Bishop JY, Lo IK, Flatow EL. Fatty infiltration and atrophy of the rotator cuff do not improve after rotator cuff repair and correlate with poor functional outcome. Am J Sports Med. 2007;35(5):719-28. 